\title{
Biochemical and Structural Analysis of Corvina (Micropogonias furnieri) Otoliths
}

\author{
M.M.M. Oliveira ${ }^{*}$, K.M. dos S. Cabral ${ }^{* *}$, E. Kurtenbach ${ }^{* *}$ and M. Farina ${ }^{*}$ \\ *Laboratório de Biomineralização, ${ }^{* *}$ Departamento de Bioquímica Médica, ${ }^{*, * *}$ Instituto de Ciências \\ Biomédicas, Universidade Federal do Rio de Janeiro, Rio de Janeiro, 21941-590, Brazil
}

The inner ear of vertebrates presents calcareous structures known as otoliths (fish) and otoconia (amphibians, reptiles, birds and mammals) that participate in the mechano-electrical transduction mechanism related to the perception of linear acceleration and gravity. In teleost fish the otolithic organs sacculus, utriculus and lagena, contain single otoliths called sagitta, lapillus and asteriscus, repectively, embedded in the endolymph [1]. It has been shown that otoliths present daily microincrements, narrow opaque zones which are taught to be annual [2]. The daily growth increments comprise an accretion zone composed mainly by crystallites of the calcium carbonate aragonite, and a discontinuous zone associated to the deposition of an organic matrix [1]. It is believed that the organic matrix play a role in otolith growth and possibly in the determination of its morphology. Until now, works on isolation of the organic matrix from otoliths use the sagitta as the object of study, probably because this is the largest ear stone in most of the teleosts.

The rational of this work is the characterization of the soluble proteins present in the organic matrix of sacule, utricule and lagena from the fish Corvina (Micropogonias furnieri). Our goal is to understand the control of shape and mineral content, as in most fishes, polymorphs of aragonite are present in the sacule and utricule while a different calcium carbonate mineral (vaterite) is present in lagenar otolith.

For biochemical analysis, otoliths were removed, washed and grounded to powder. The sample was dialyzed against $10 \%$ EDTA, $\mathrm{pH} 7$ until complete decalcification. The entire extract was then centrifuged and the supernatant (otolith soluble-matrix - OSM) was subjected to biochemical analysis. Structural analysis was performed using transmission (TEM) and scanning electron microscopy (SEM). TEM samples were prepared as follows: small pieces of the otoliths were cut, demineralized/fixed with an EDTA/glutaraldehide containing solution, included in epoxy resin, ultrathin sectioned with diamond knife, and stained with uranil acetate and lead citrate. SEM samples were obtained by polishing sections cut perpendicularly both to the major axis of the otolith and the surface near the sensory epithelium. Observation of the samples was done in a JEOL 1200 EX (TEM) and a JEOL 5310 (SEM).

SDS-PAGE of the soluble- matrix from sacular otolith presented mainly three protein bands of 107 , 77.5 and $56.9 \mathrm{kDa}$. The 107 and $56.9 \mathrm{kDa}$ protein bands were positive to PAS and Alcian Blue reactions indicating that they were glycosilated. These proteins were calcium binding as intense radioactivity was obtained in the corresponding bands when assay with ${ }^{45} \mathrm{Ca}$ was performed. Preliminary results using the soluble-matrices of utricule and lagena otoliths show a major protein band of ca. $77 \mathrm{kDa}$ for both samples.

TEM images of decalcified otolith showed highly stained bands with varying distances one to the other, and thin filaments running perpendicularly to the previous bands regularly spaced in the sagitta (Fig. 1). When compared to polished section observed by SEM (Fig. 2), it was concluded that the highly stained regions in Fig. 1 represent the organic matrix of the discontinuous zone, while the thin filaments disposed perpendicularly, would correspond to the organic matrix in between (or inside) the aragonite crystals. Fig. 2 shows that the longer axis of the aragonite crystallites align in a direction perpendicular to the growth lines. Lagena otoliths also presented growth lines (Fig. 3); however crystallites seemed to be smaller and with no observable orientation. Also the organic matrix region appeared larger than in sagitta, and trabecular structures linking mineralized blocks disposed perpendicular to growth lines, 
were visible (Fig. 3). Fractured surfaces of utricular otolith presented a lower number of growth lines when compared to the previous ones. These areas show lines closely spaced (Fig. 4).

Further studies on the composition of otolith organic matrix and endolimph will provide information about the differences between the mineral phases of the calcium carbonates found in the sacule and utricle (aragonite), and that of lagena (vaterite). Morphological evaluation of the ear stones performed in this work showed that otoliths from the three organs present growth lines, although different patterns were observed, reflecting different proportions of mineral/organic matrix contents.

\section{References:}

[1] Y. Takagi and A. Takahashi. The Anatomical Record 254 (1999) 322.

[2] R.W. Gauldie and R.L. Radtke. Comp. Biochem. Physiol. 974 (1990) 137.

[3] Financial Support: CNPq and Faperj Brazilian Agencies.
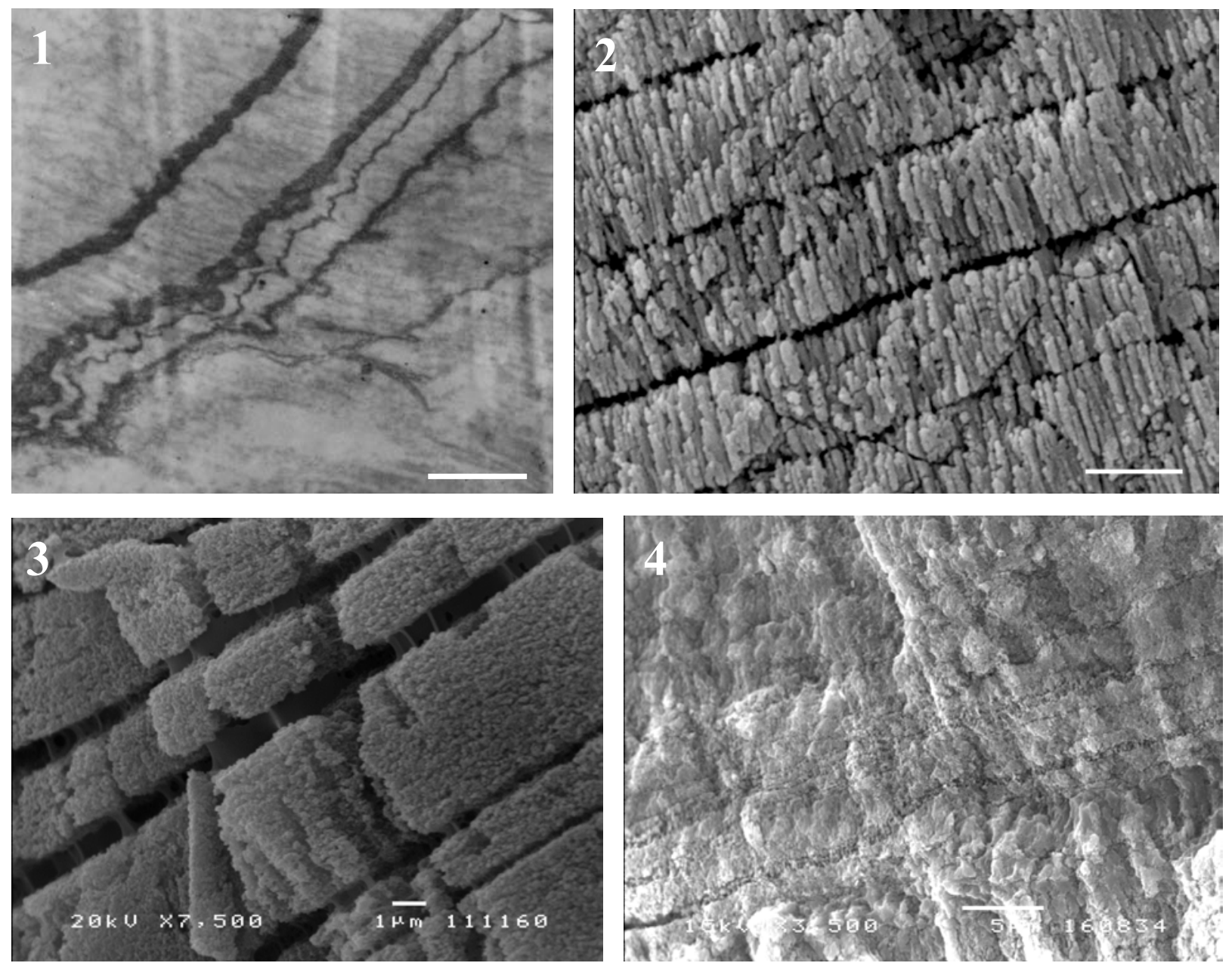

Figure 1: Ultrathin section of decalcified sagitta showing the organic region parallel to growth lines, and the thin filaments. TEM image. Bar $=2 \mu \mathrm{m}$.

Figure 2: Polished section of sagitta showing aragonite crystals aligned perpendicular to the growth lines. SEM image. Bar $=2 \mu \mathrm{m}$.

Figure 3: Polished section of asteriscus. Note growth lines and trabeculae. SEM image. Bar $=1 \mu \mathrm{m}$. Figure 4: Fractured surface of lapillus showing closely spaced growth lines. Bar $=5 \mu \mathrm{m}$. 